\title{
Path planning and kinematics simulation of surfacing cladding for hot forging die
}

\author{
Huajun Wang ${ }^{1, a}$, Wanxia $\mathrm{Fu}^{1}$, Hengan $\mathrm{Ou}^{2}$, and Xuan Tang ${ }^{3}$ \\ ${ }^{1}$ Wuhan University of Technology, School of Materials Science and Engineering, \\ Wuhan 430070, China \\ ${ }^{2}$ Department of Mechanical, Materials and Manufacturing Engineering, University of Nottingham, \\ Nottingham NG7 2RD, UK \\ ${ }^{3}$ Dongfeng Motor Co., Ltd, Wuhan 430070, China
}

\begin{abstract}
During the course of their work, a variety of damage and failure of hot forging die occurs and seriously affect the service life. Multi-layer metal hot forging die with functionally graded material structure can effectively extend the service life. In this paper, According to the needs of strengthening forging cavity, the CAD model of surfacing forming center was designed. Based on technological requirements of surface cladding for die cavity, the coupled movement equation of weld torch was established, and the trajectory of welding positioner and Cartesian robot kinematics was solved. The weld torch path was planned, according to the typical methods used in plane welding, and the surfacing path data was extracted by the secondary development of UG/OPEN. Then the kinematics solver program, which can output the control function of motion simulation, was written in MATLAB to solve the kinematics equation. Finally, in UG NX7.5, the kinematics simulation model was built to verify the correctness of mathematical model and the rationality of welding path planning. The above studies can provide a technical support for the die repair and manufacturing of a multilayer metal forging die.
\end{abstract}

\section{Introduction}

Hot forging die is an important technological equipment of manufacturing and processing industry. In hot forging operations, the die surface and the nearest surface layers are subjected to coupled mechanical and thermal cycles which significantly influence their service life. Die failure is caused by different phenomena: wear, plastic deformation and thermo-mechanical fatigue [1,2]. Therefore, a series of researches have been started to prolong the life of hot forging die. Plasma arc welding Surfacing (PAWS) has been developed to overlap metal cladding in die surface layer. The cladding layer has high mechanical strength and heat-resistant performance, so it can meet the needs of the deformation of workpiece and prevent high temperature creep distortion [3]. Experiments and simulations proved that

\footnotetext{
${ }^{\text {a }}$ Corresponding author: wanghuajunhb@163.com
}

This is an Open Access article distributed under the terms of the Creative Commons Attribution License 4.0, which permits unrestricted use, distribution, and reproduction in any medium, provided the original work is properly cited. 

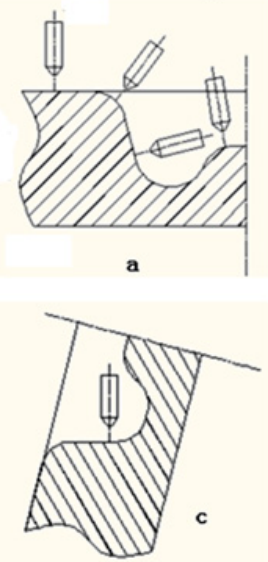
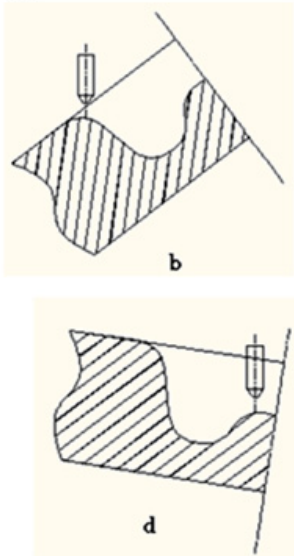

Figure 1. Space curve surfacing welding seam position: b, c, d are ideal position.

multilayer metal die with functionally graded materials structure can effectively extend the service life, which is manufactured through PAWS method $[4,5]$.

Considering the forging cavity being non-planar structure and the coating being accumulated by space curve welding seam, the welding seam only has a short period in the ideal position of level/ship. Fig. 1 shows that the welding torch must keep moving to ensure the welding seam at the level/ship position, which is essential to obtain better shape and weld quality and maintain the continuity of welding process [6]. According to the non-planar structure of hot forging die, surfacing welding center was designed. At present, PAWS system is at a low degree of automation control, the existing equipment and production technology cannot meet their manufacturing requirements. Cladding welding technology of the complex surfaces still rely on manual welding technology, which mean the poor working conditions for workers and the poor welding quality and low efficiency. So it is necessary to developed automation surfacing technology [7].

On the basis of the welding characteristics of the multilayered metal forging die, a mathematical model and a kinematics simulation model of a five-DOF arm CNC forming center were established in this paper. The kinematics simulation model was used to simulate the surfacing process, and the results were used to verify the correctness of mathematical model and the rationality of welding path planning $[8,9]$. This study will provide a theory foundation to develop PAWS system and a solution to manufacture multilayer metal forging die. So it has great value of application and development potential in metal forming tools.

\section{5-axis arm-type surfacing forming centre}

The movement mechanism of 5-axis arm-type surfacing forming centre is built up with two-freedomdegree for rotational motions of welding positioner and three-freedom-degree of Cartesian robot. In the welding process, the weld seam is required at level/ship position, while the torch should be mounted vertically relative to level/ship line and maintains a certain distance from the weld surface. The welding positioner can rotate around its own axis and swing with a constant angular velocity to complete the welding process, while the robot moves in $\mathrm{x}, \mathrm{y}$ and $\mathrm{z}$-axis direction to determine the position of the torch. 


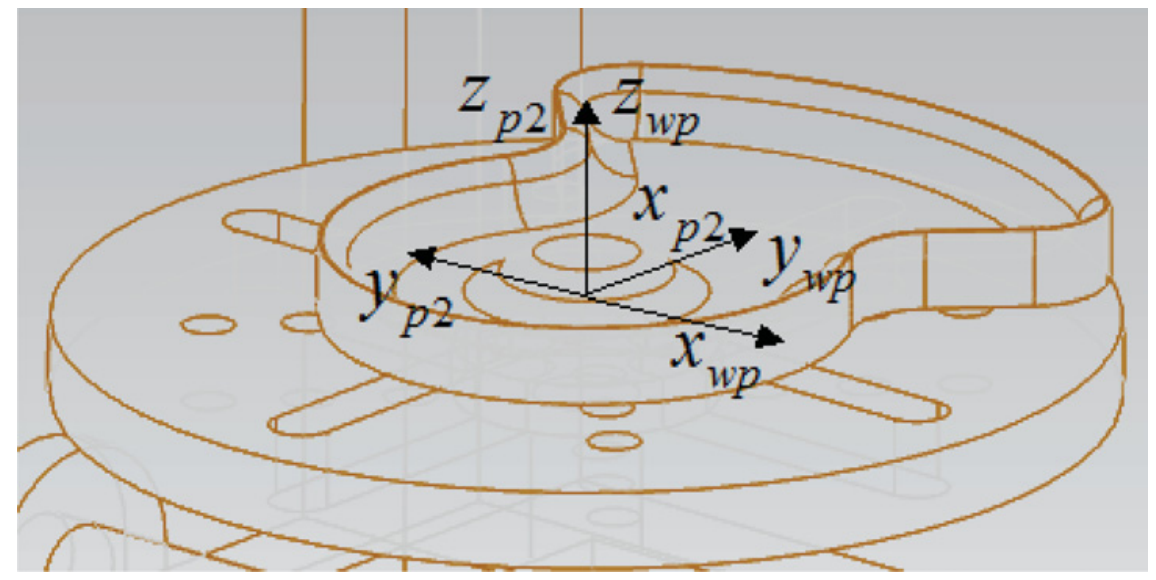

Figure 2. Workpiece and workbench coordinate.

\subsection{The mathematical model between welding positioner and workbench}

During the operation of the surfacing forming center, the workpieces was fixed on the welding positioner, which provided two-freedom-degree to ensure the weld seam at level/ship position. Figure 2 precisely described the location of the weld seam in the workspace. The weld seam coordinate transformation matrix from processing center system into world coordinate system was obtained:

$$
{ }_{\text {work }}^{w} T=\left[\begin{array}{cc}
n_{x} \cos \beta-n_{y} \sin \beta & \sigma_{x} \cos \beta-o_{y} \sin \beta \\
n_{x} \cos \alpha \sin \beta+n_{y} \cos \alpha \cos \beta-n_{z} \sin \alpha & o_{x} \cos \alpha \sin \beta+o_{y} \cos \alpha \cos \beta-o_{z} \sin \alpha \\
n_{x} \sin \alpha \sin \beta+n_{y} \cos \alpha \cos \beta+n_{z} \cos \alpha & o_{x} \sin \alpha \sin \beta+o_{y} \sin \alpha \cos \beta+o_{z} \cos \alpha \\
0 & 0
\end{array}\right.
$$

$$
a_{x} \cos \beta-a_{y} \sin \beta \quad p_{x} \cos \beta-p_{y} \sin \beta
$$

$a_{x} \cos \alpha \sin \beta+a_{y} \cos \alpha \cos \beta-a_{z} \sin \alpha \quad p_{x} \cos \alpha \sin \beta+p_{y} \cos \alpha \cos \beta-\left(p_{z}+160\right) \sin \alpha$

$a_{x} \sin \alpha \sin \beta+a_{y} \cos \alpha \cos \beta+a_{z} \cos \alpha \quad p_{x} \sin \alpha \sin \beta+p_{y} \sin \alpha \cos \beta+\left(p_{z}+160\right) \cos \alpha+250$

When the welding seam at level/ship position the following equation is established:

$$
\left\{\begin{array}{l}
a_{x} \cos \beta-a_{y} \sin \beta=0 \\
a_{x} \cos \alpha \sin \beta+a_{y} \cos \alpha \cos \beta-a_{z} \sin \alpha=0 \\
a_{x} \sin \alpha \sin \beta+a_{y} \sin \alpha \cos \beta-a_{z} \cos \alpha=1 .
\end{array}\right.
$$

The value of ax, ay, az was extracted from secondary development of UG/OPEN, which the flip angle $\alpha$ and rotation angle $\beta$ can be calculated.

\subsection{The mathematical model between Cartesian robot and welding torch}

Cartesian robot, where the welding torch is fixed on, is moving in $\mathrm{x}, \mathrm{y}$ and $\mathrm{z}$-axis direction to maintain the continuity of the surfacing process. The pose relation between the welding torch and seam is shown 


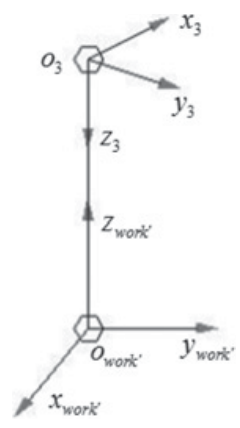

Figure 3. Position and orientation relation between welding torch and welding seam

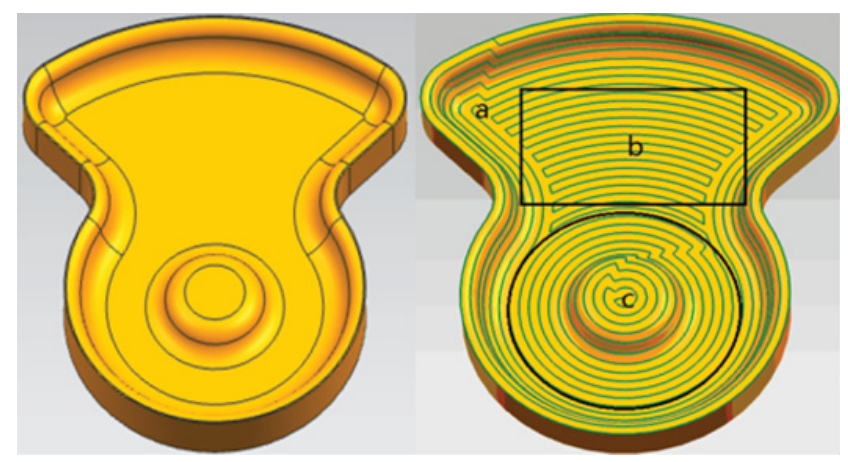

Figure 4. Surfacing partition and Surfacing path planning of an instance.

in Fig. 3, then position and orientation of welding seam coordinates was obtained in the world coordinate system. The origin position of the welding seam and torch coordinate are shown in (3) and (4).

$$
\begin{gathered}
p_{x} \cos \beta-p_{y} \sin \beta \\
{\left[\begin{array}{c}
p_{x} \cos \alpha \sin \beta+p_{y} \cos \alpha \cos \beta-\left(p_{z}+160\right) \sin \alpha \\
p_{x} \sin \alpha \sin \beta+p_{y} \sin \alpha \cos \beta+\left(p_{z}+160\right) \cos \alpha+250 \\
1
\end{array}\right]} \\
p_{x} \cos \beta-p_{y} \sin \beta=\alpha \\
p_{x} \cos \alpha \sin \beta+p_{y} \cos \alpha \cos \beta-\left(p_{z}+160\right) \sin \alpha=b \\
p_{x} \sin \alpha \sin \beta+p_{y} \sin \alpha \cos \beta+\left(p_{z}+160\right) \cos \alpha+250+d_{\text {weld }}=750+c .
\end{gathered}
$$

Then the vaule of $\mathrm{a}, \mathrm{b}$ and $\mathrm{c}$ can be calculated.

\section{Path planning of surfacing}

The instance part has the typical geometry feature of hot forging die, which include flat and non-planar structure. Due to the special structure of the instance part, contour and parallel scanning method was used to design the welding path.

As shown in Fig. 4, the part surface is divided into three regions, with different scanning mode: a) peripheral concave wall, using contour-parallel paths; b) fan-shaped, using square parallel paths; 


\section{ICNFT 2015}
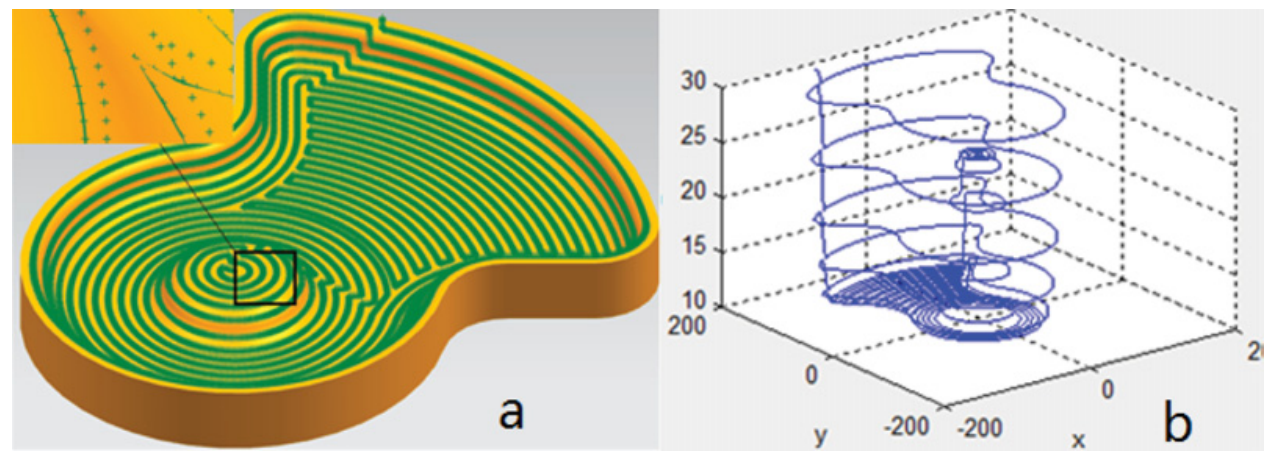

Figure 5. The 3-D graphics of instance part's trajectory (a) The discrete solder joints of surfacing path and the corresponding normal vector; (b) The three-dimensional graphics of instance part's trajectory.

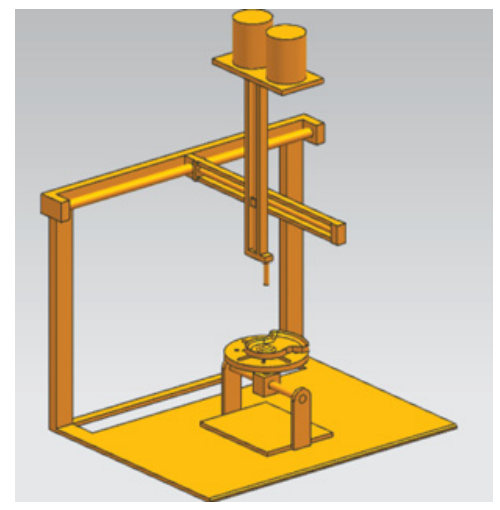

Figure 6. The simplified movement model of surfacing welding centre.

c) internal circular spigot area, using contour-parallel paths. The weld width of plasma transferred arc welding is generally $2-13 \mathrm{~mm}$, tentatively scheduled for weld pitch $6 \mathrm{~mm}$. After determining the partition and paths, surfacing paths are drawn with UG NX7.5.

According to surfacing planning path, the surfacing path data was extracted and the normal vector of solder joint was written by the secondary development of UG/OPEN. As shown in Fig. 5, the solder joints and corresponding normal vector was scattered and saved to the appointed path.

\section{The kinematics simulation of welding path}

As shown in Fig. 6, the surfacing equipment was established and assembled into a virtual whole machine using the software UG NX7.5. Then the virtual assembled machine was checked and optimized.

In the automatic welding process, through the UG/OPEN GRIP programming, the surfacing path curve become a series of discrete points and the kinematic parameters of these points were compiled and computed by MATLAB. Then the position data of the arm robot and the angle data of the welding positioner were output. Through kinematics simulation, we could observe that the solder joints were moved to level/ship position by flipping and rotating of the welding positioner during operation, while the welding torch was operated by Cartesian robot to track the joints. Figure 7 shows the operating parameters for the torch and the positioner at the start time, at the 2090s, at the 9096s and the end time of the simulation. 


\section{MATEC Web of Conferences}
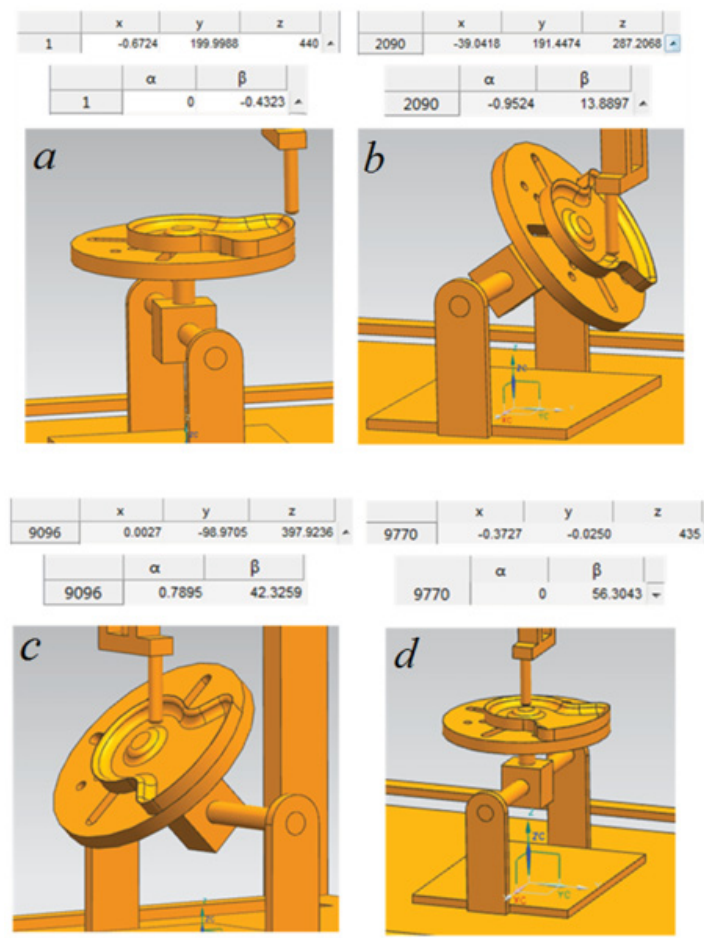

Figure 7. The process of kinematic simulation: (a) at 1s; (b) at 2090s; (c) at 9096s; (d) at 9770s.

Through motion simulation of the instance parts, we can deduce that the driving movements could meet the requirements of the surfacing welding and the interference between the torch and positioner did not appear in the working process, which indicated that the planning path was feasible.

\section{Conclusions}

(1) Design method of plane path planning in the rapid prototyping process is introduced into the surface welding path planning, and surfacing path of instance part was planned.

(2) Kinematics simulation model of the surfacing welding centre was established with UGNX to simulate the welding path of instance part. The results verified that the surfacing path planning could be effectively applied in the surface cladding technology of instance part and the offline programming system about motion simulation functions was realized.

(3) By the path planning and kinematics simulation of surfacing forming centre, the difficulty of automatic surfacing process for hot forging die was solved and the development of technology and equipment about the plasma arc welding process could be technically supported.

The authors are grateful to the Nation Natural Science Foundation of China (5145346) and the Fundamental Research Funds for the Central Universities (2010-II-025) for financial support of this work. 


\section{ICNFT 2015}

\section{References}

[1] H. J. Wang, Y. Z. Wu, H. C. Wang, Y. Z. Sun, G. Wang. Materials Research Innovations, 15(s1): 377-380 (2011)

[2] M. Bayramoglu, H. Polat, N. Geren. Journal of Materials Processing Technology, 205 (2008)

[3] C. Pelz. Materials Science Forium, 308-311(8): 634-640 (1999)

[4] S. Suryakumar, K.P. Karunakaran, A. Bernard, U. Chandrasekhar, N. Raghavender, Deepak Sharma. Computer-Aided Design, 43(4): 331-344 (2011)

[5] B.A. Behrens, F. Schaefer. Journal of Materials Processing Technology, 167: 309-315 (2005)

[6] Wang H J, Zhang Y, Lu J F, Tang X. Automatic plasma arc welding equipment for hot forging Cavity cladding [P]. China, ZL201320402806.4

[7] M. Jouaneh, D. Dornfeld. Journal of Manufacturing Systems. 7(4): 307-314 (1988)

[8] George-Christopher Vosniakos, Zenon Kannas. Robotics and Computer-Integrated Manufacturing, 25(2): 417-431 (2009)

[9] X. Shan, C.M. Davies, T. Wangshan, et al. International Journal of Pressure Vessels and Piping, 86(1): 110-121 (2009) 